High voltage multiplexing for the ATLAS Tracker Upgrade

This content has been downloaded from IOPscience. Please scroll down to see the full text. 2014 JINST 9 C01032

(http://iopscience.iop.org/1748-0221/9/01/C01032)

View the table of contents for this issue, or go to the journal homepage for more

Download details:

IP Address: 131.169.3.59

This content was downloaded on 19/01/2015 at 11:02

Please note that terms and conditions apply. 


\title{
High voltage multiplexing for the ATLAS Tracker Upgrade
}

\author{
E.G. Villani, ${ }^{a}{ }^{1}$ P. Phillips, ${ }^{a}$ J. Matheson, ${ }^{a}$ D. Lynn, ${ }^{b}$ L.B.A. Hommels, ${ }^{c}$ I. Gregor, ${ }^{d}$ \\ M. Bessner, ${ }^{d}$ K. Tackmann, ${ }^{d}$ F.M. Newcomer, ${ }^{e}$ E. Spencer ${ }^{f}$ and A. Greenall ${ }^{g}$ \\ ${ }^{a}$ STFC Rutherford Appleton Laboratory Particle Physics Dept., \\ Harwell, Didcot, OX110QX, U.K. \\ ${ }^{b}$ Brookhaven National Laboratory (BNL), \\ Upton (NY), U.S.A. \\ ${ }^{c}$ Cavendish Laboratory, University of Cambridge, \\ Cambridge, U.K. \\ ${ }^{d}$ Deutsches Elektronen Synchrotron (DESY), \\ Hamburg, Germany \\ ${ }^{e}$ University of Pennsylvania, \\ Philadelphia (PA), U.S.A. \\ ${ }^{f}$ University of California Santa Cruz (SCIPP-UCSC), \\ Santa Cruz, (CA), U.S.A. \\ ${ }^{g}$ Oliver Lodge Laboratory, University of Liverpool, \\ Liverpool, U.K.
}

E-mail: giulio.villani@stfc.ac.uk

ABSTRACT: The increased luminosity of the HL-LHC will require more channels in the upgraded ATLAS Tracker, as a result of the finer detector segmentation, stemming from the otherwise too high occupancy. Among the many technological challenges facing the ATLAS Tracker Upgrade there is more an efficient power distribution and HV biasing of the sensors. The solution adopted in the current ATLAS detector uses one HV conductor for each sensor, which makes it easy to disable malfunctioning sensors without affecting the others, but space constraints and material budget considerations renders this approach impractical for the Upgraded detector. A number of approaches, including the use of the same HV line to bias several sensors and suitable HV switches, along with their control circuitry, are currently being investigated for this purpose. The proposed solutions along with latest test results and measurements will be described.

KEYWORDS: Voltage distributions; Data acquisition circuits; Radiation-hard electronics; Large detector systems for particle and astroparticle physics

\footnotetext{
${ }^{1}$ Corresponding author.
} 


\section{Contents}

1 Introduction 1

2 HV MUX description 2

2.1 Devices description and tests 3

2.2 Control board description and test 5

3 Conclusions 6

\section{Introduction}

In the Semiconductor Tracker system (SCT), one of the three sub-detectors of the present ATLAS tracker, the High Voltage biasing to the strip sensors is provided by individual High Voltage supplies, remotely located in a service cavern [1,2].

To have one HV conductor for each sensor represents an optimal solution in terms of robustness, as this simplifies the operation of disabling malfunctioning sensors without disturbing the others. Also it allows easy monitoring of the individual current drawn by each sensor, an important requisite to assess their proper functioning.

The ATLAS Tracker Upgrade will consist of an all silicon tracking detector, to fulfill the requirements of the High Luminosity upgrade of the Large Hadron Collider (HL-LHC) [3, 4]. The baseline solution for the strip barrel is the stave concept, in which a number, up to 13, of single sided silicon strip detector modules are glued directly to each side of a composite support carbon fibre structure, which embeds the cooling pipe, figure 1. The routing of signals and power between the modules and the End of Stave (EoS) board, that acts as interface to the stave and is located at one end of the stave, is provided by a long kapton flex circuit, known as Bus Tapes, that is glued to the carbon fibre skin of the stave.

Owing to the increased number of sensors in the Tracker Upgrade it will be not feasible to use the existing HV conductors to implement the individual biasing approach: lack of space for cables and traces will not permit each sensor to have its own HV bias [5]. For example, if existing cables are re-used, groups of 4 sensors or more will need to be connected in parallel and this would lead to the loss of the other modules on the same bias line, should one sensor fail due to high current. To avoid such potential losses, the approach currently being investigated consists of having all the 13 modules on one side of the stave powered by a single line. Each sensor can be disconnected from the bias line with a DCS controlled HV multiplexer switch (MUX). A detailed description of the proposed HV MUX approach, along with tests results of investigated HV switches and related control board, will be provided on section 2 . 


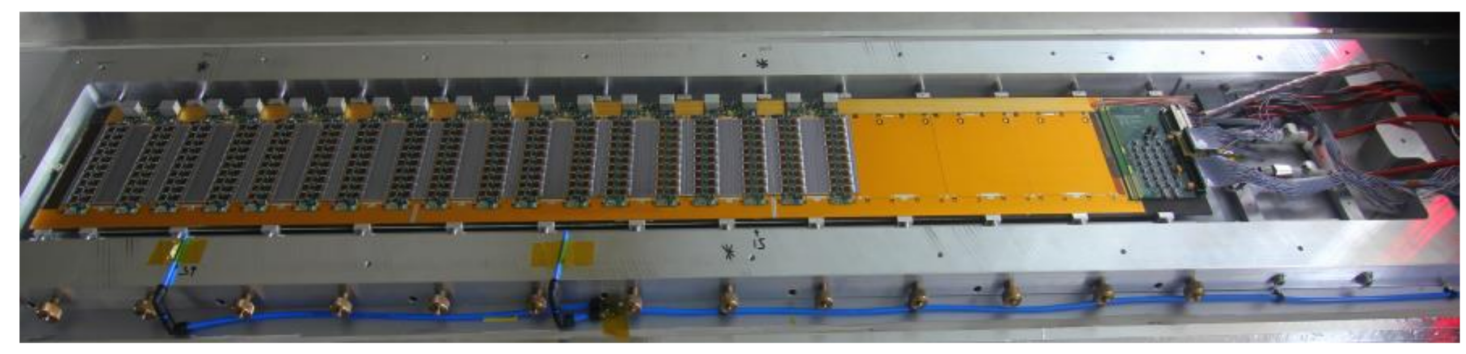

Figure 1. A stave being assembled at RAL.

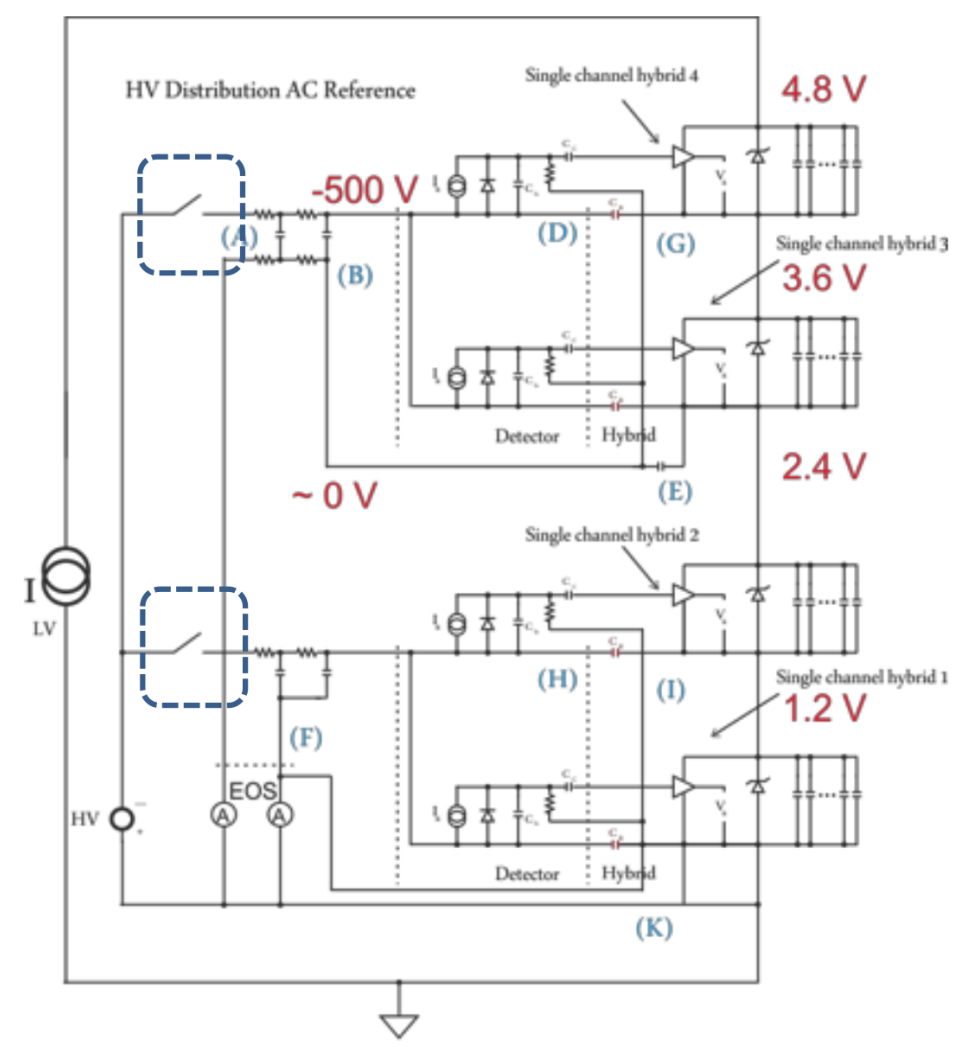

Figure 2. A simplified HV MUX schematic diagram in a SP scheme. The HV switches, within the dashed frame in the picture, share the common low side of the HV bias.

\section{HV MUX description}

The schematic diagram of the HV MUX approach is shown in figure 2 with reference to a serial powering scheme, but it would be equally valid for a DC to DC powering scheme. Each HV switch is individually addressable through DCS signal and permits enabling or disabling of the HV biasing to a sensor.

When in off mode, the HV switches should be able to withstand in excess of $500 \mathrm{~V}$, at the same time exhibiting much smaller leakage current than the sensors operating under normal condition (few 100's nA when non irradiated). Conversely, when in the on mode they should present a relatively low resistance, to allow the flow of at least a few mA's, owing to the increased leak- 


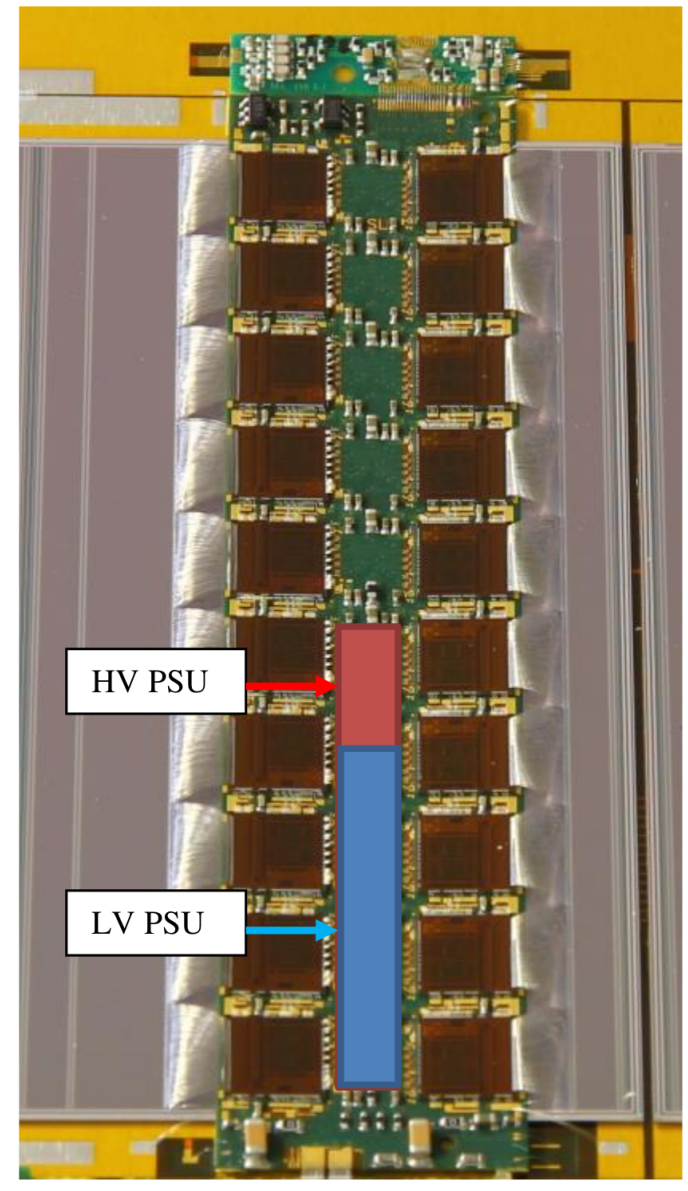

Figure 3. Proposed allocated area, of approximately $6 \times 40 \mathrm{~mm}^{2}$, for the HV MUX on stave hybrid.

age of the sensors after years of exposure to radiation. The predictions for the maximum fluence and dose in the strip barrel detectors are $5.3 \cdot 10^{14} 1 \mathrm{MeV} \mathrm{n}$ eq $/ \mathrm{cm}^{2}, 216 \mathrm{kGy}$ for the short strips in layer 1 and $8.1 \cdot 10^{14} 1 \mathrm{MeV} \mathrm{n}_{\mathrm{eq}} / \mathrm{cm}^{2}, 288 \mathrm{kGy}$ for the strip end cap [6]. A specification of $2 \cdot 10^{15} 1 \mathrm{MeV} \mathrm{n}$ eq $/ \mathrm{cm}^{2}$ is therefore imposed to allow for uncertainties in fluence calculations, which sets the targeted radiation tolerance of the HV switches. Moreover, the HV switches should be able to operate in a strong magnetic field of around $2 \mathrm{~T}$, at low temperature and to be economically viable, as the estimated number of devices needed is in excess of 10,000.

The proposed allocated area for the implementation of the HV MUX is on the hybrid of the strip module, figure 3, where a small PCB carrier would include the LV powering, the HV MUX and the current measuring circuitry.

\subsection{Devices description and tests}

The very stringent requirements for the HV switches outlined above make it difficult to identify readily available silicon devices suitable for this application. A number of devices, based upon silicon and wider band gap material, have been thoroughly investigated to assess their suitability to the task. 


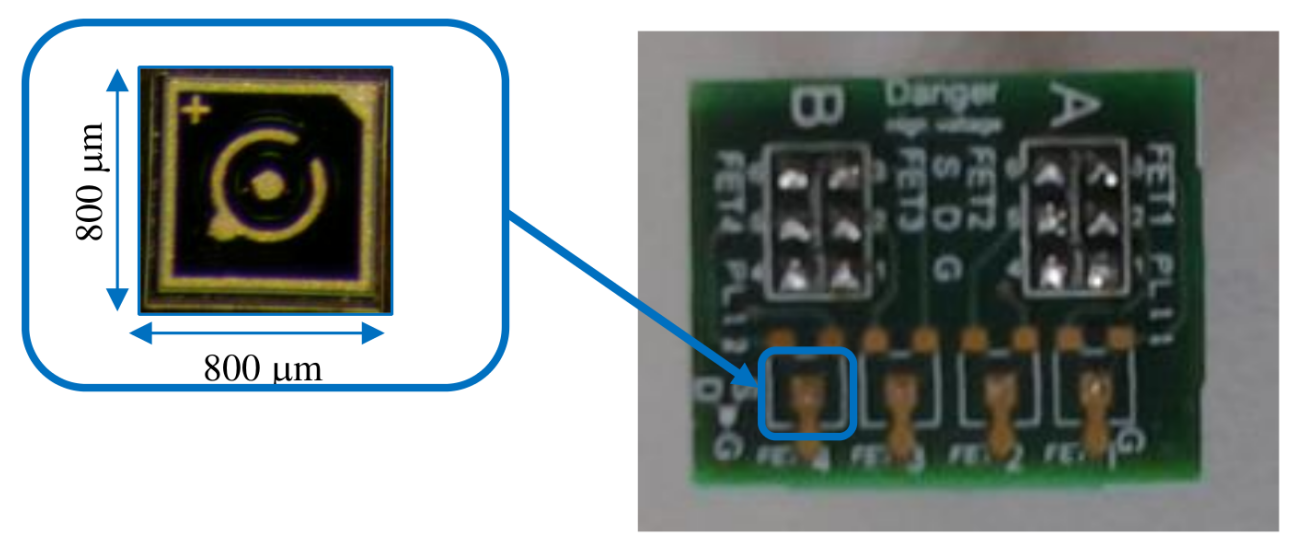

Figure 4. A silicon JFET 2N6449 die picture. Four devices were glued and wire bonded on a PCB carrier for testing.

The detrimental effects of ionizing radiation on standard silicon based MOSFET are well known and include threshold voltage shift, increase in leakage current and reduction in breakdown voltage [7]. However, owing also to the lack of gate oxide, standard silicon devices JFET could potentially be less sensitive to both ionization and displacement effects $[8,9]$. For this project, a number of $\mathrm{n}$ type silicon JFETs rated for high voltage has been investigated. In figure 4 a picture of a silicon $\mathrm{N}$ type JFET $2 \mathrm{~N} 6449$ is shown. Despite being rated for a $V_{\mathrm{ds}}$ maximum of $300 \mathrm{~V}$, which is lower than what needed for this project, simulations showed the possibility of using a configuration of stacked devices that would allow higher voltage operation.

After an initial electrical characterization at RAL, using a Keithley CS 4200 semiconductor parameter analyzer, a number of such devices were irradiated, unbiased, using protons beam at $26 \mathrm{MeV}$ at the University of Birmingham, U.K. and characterized again to investigate radiation effects on their characteristics. The estimated fluence was $10^{15} 1 \mathrm{MeV} \mathrm{n}_{\mathrm{eq}} / \mathrm{cm}^{2}$ with a total dose of radiation delivered of $0.9 \mathrm{MGy}$.

In figure 5 some of their electrical characteristics before and after irradiation are shown. An increased leakage current when in the off state is observed, that would nonetheless be tolerable when compared to expected increase leakage of the sensor. However, the increase in on state resistance, believed to be due to the $\mathrm{n}$ type bulk radiation damage, would not allow currents of some $\mathrm{mA}$ to flow through the devices for proper biasing of the sensors.

Additional tests on HV rated silicon devices are planned but alternative technologies, based upon wide band gap materials, like $\mathrm{SiC}$ and $\mathrm{GaN}$, are also being investigated, by virtue of their expected superior performances in terms of $\mathrm{HV}$ and radiation hardness [10]. In figure 6 a picture of a test HV MUX based on a SiC Power JFET SJEP170R550 is shown, along with its schematic diagram. The designed MUX consists of 4 channels and has been successfully tested to verify that no additional noise is introduced to the sensors signal path by these devices. Subsequent irradiation tests performed on these devices showed no appreciable signs of radiation damage. Unfortunately, the manufacturer went out of business in 2012 and it is not clear how the availability of these devices will be in the long term [11].

Other characterizations have been performed on SiC JFET and MOSFET, further radiation tests will be carried out soon. 

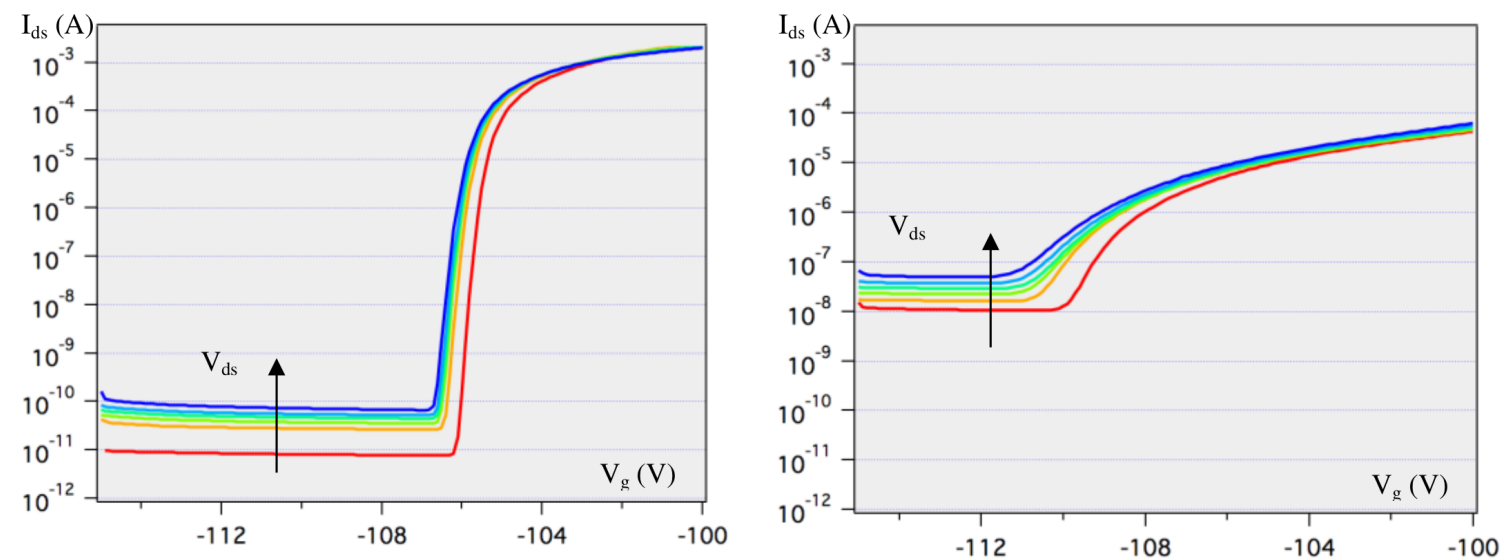

Figure 5. An example of $I_{\mathrm{ds}}$ vs. $V_{g}$ curves for non-irradiated (left) and irradiated (right) HV silicon JFET 2N6449. A complete shut off is achieved for $V_{\mathrm{gs}}$ below $-12 \mathrm{~V}$. The $V_{\mathrm{ds}}$ varied between $[-100,+185] \mathrm{V}, V_{g}$ between $[-115,-100] \mathrm{V}] \mathrm{V}$ and source kept at $V_{s}=-100 \mathrm{~V}$. Maximum $I_{\mathrm{ds}}$ compliance $2 \mathrm{~mA}$. Following irradiation, the leakage current in off state increases by around 3 orders of magnitude, the rdson in on state by almost 2 .
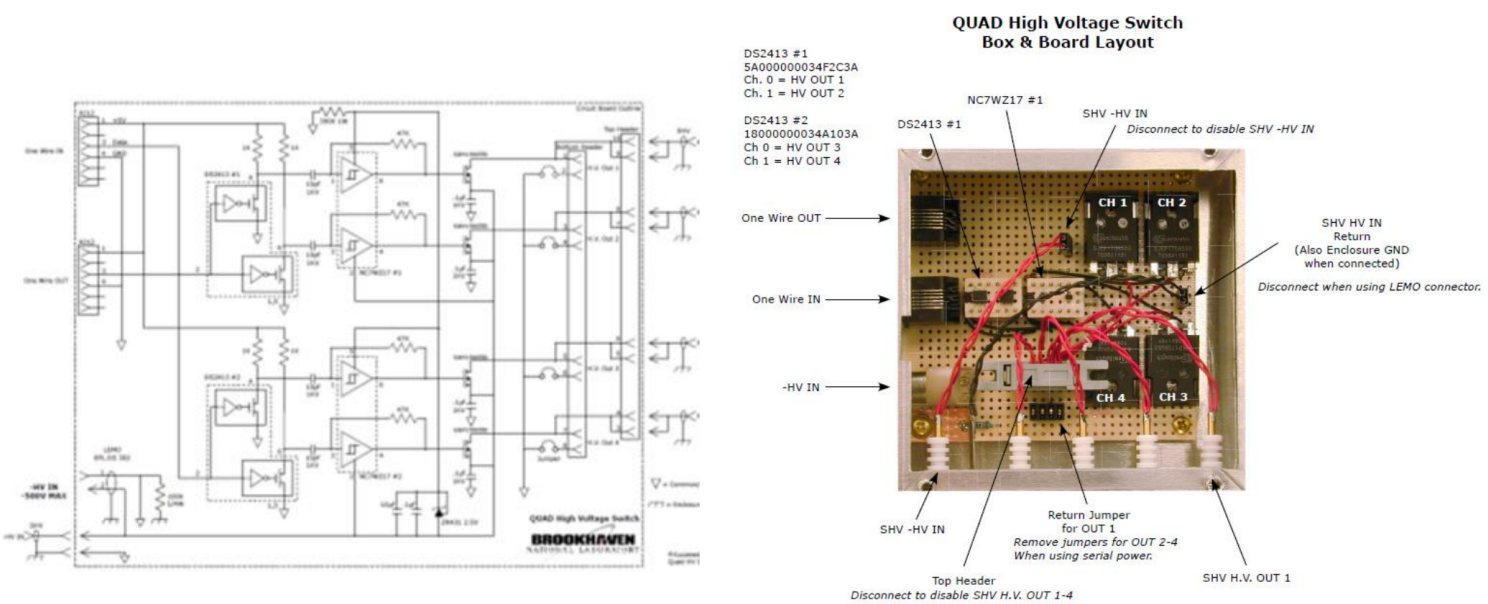

Figure 6. A 4 channels HV MUX based on SiC JFET SJEP170 used for preliminary tests on stavelets (reduced versions of staves). Irradiation tests carried out on these devices showed negligible evidence of degradation in their electrical performances.

\subsection{Control board description and test}

Regardless of the specific HV devices that will be used in the HV MUX, a control circuitry to enable or disable them via a low voltage DCS signal will be needed. The current plan is to implement such control circuitry on the same carrier board holding the HV MUX.

A possible circuit topology currently being investigated is shown in figure 7. An AC coupled voltage multiplier generates a negative (or positive, depending on the diodes orientation) voltage output, of adequate amplitude to turn on or off the HV devices. The input signal consists of a low frequency square wave signal $(50 \mathrm{kHz}$ in the example being shown), generated by DCS circuitry. The output voltage amplitude depends on the number of multiplying stages and the output current 


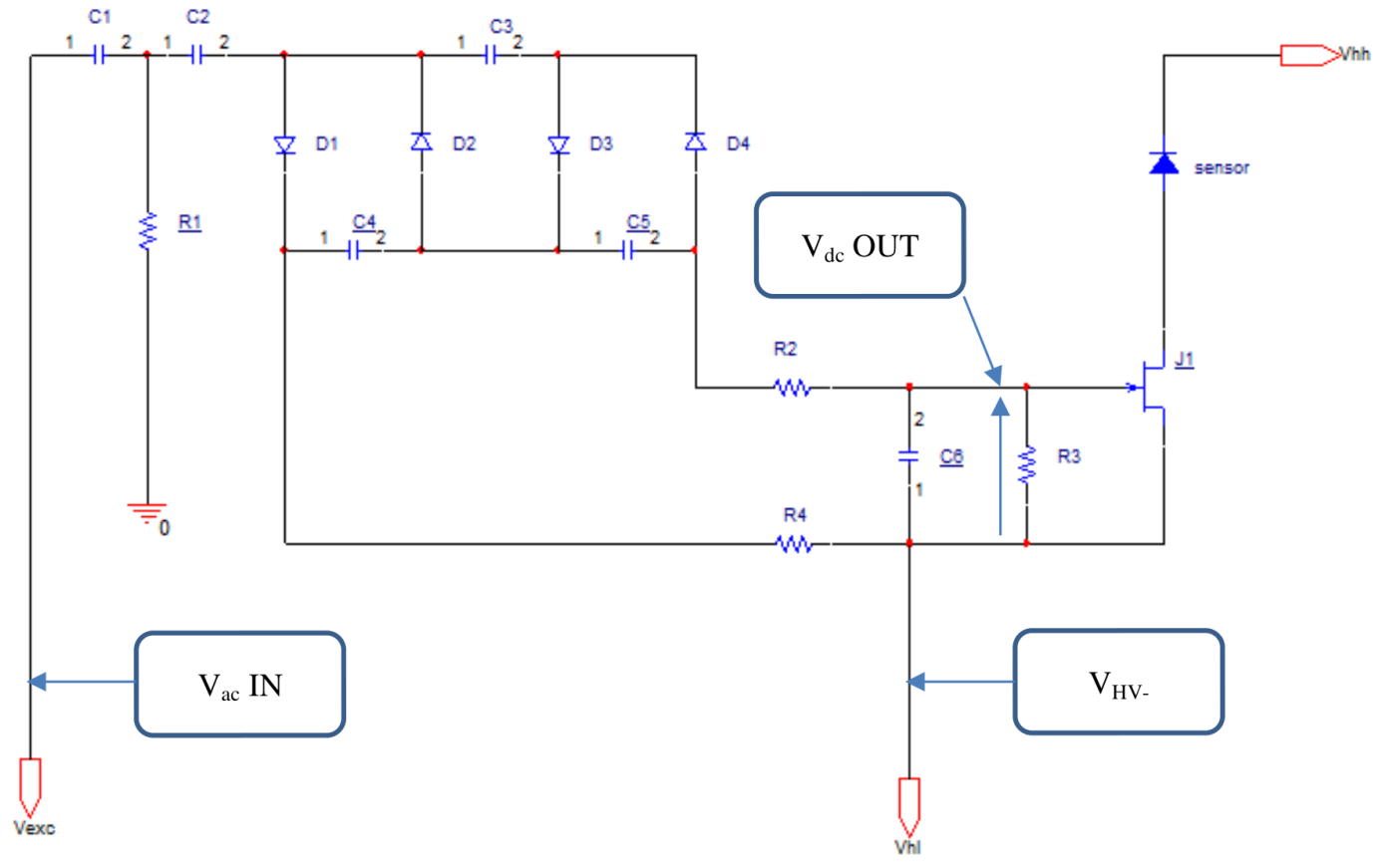

Figure 7. A schematic diagram of an AC coupled voltage multiplier for the enable of the HV switches. The sign of the output voltage depends on the diodes orientation (negative in the example shown), its amplitude on the number of stages, the forward drop of the diodes used and the current drawn by the load.
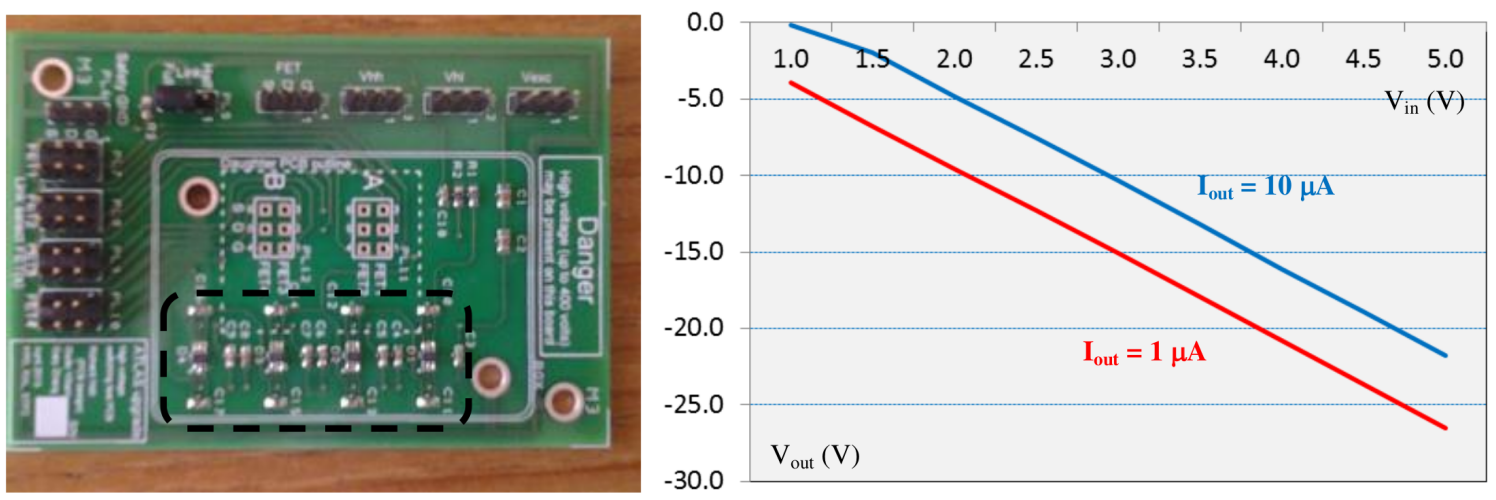

Figure 8. An example of negative voltage multiplier consisting of 8 Schottky stages (within the dashed frame in the left picture). The measured output voltage vs. input voltage is shown on the right plot for two values of current output. The input voltage consists of a square wave of $50 \mathrm{kHz}, 50 \%$ duty cycle and variable amplitude.

being drawn, thus the final voltage multiplier design will depend on the specific type of HV switch chosen. In figure 8 some initial tests results are shown.

\section{Conclusions}

The HV distribution in the ATLAS Tracker upgrade will require sharing of the HV line among several sensors. In order to maintain an acceptable level of robustness each sensor will have to be 
equipped with a HV switch, to prevent impairment of the functioning of other sensors connected in parallel.

A number of HV devices, based on silicon and wider band gap materials, are currently being investigated for this purpose. Electrical and radiation tests have been performed, suggesting that a $\mathrm{SiC}$ based solution might be a viable route.

A control circuitry to allow enabling of the HV switches is also being investigated.

\section{Acknowledgments}

The authors would like to thank John Wilson, Paul Dervan and Zhige Zhang for their help in the setting up of the irradiation test at Birmingham University.

\section{References}

[1] P.W. Phillips, ATLAS SCT power supply system, in Proceedings of the Topical Workshop on Electronics for Particle Physics (TWEPP07), Prague Czech Republic (2007), CERN-2007-007, pp. 365-368.

[2] J. Böhm et al., Power supply and power distribution system for the ATLAS silicon strip detectors, in Proceedings of the Seventh Workshop on Electronics for LHC Experiments, Stockholm Sweden (2001), CERN-2001-005, pp. 363-367.

[3] A. Clark, M. Elsing and P. Wells, Performance specifications of the Tracker Phase II Upgrade, ATL-UPGRADE-PUB-2012-003 (2012).

[4] A. Clark et al., Final report: ATLAS Phase-II Tracker Upgrade layout task force, ATL-UPGRADE-PUB-2012-004 (2012).

[5] ATLAS collaboration, Letter of intent for the Phase-II Upgrade of the ATLAS experiment, CERN-LHCC-2012-022 (2012).

[6] P.S. Miyagawa and I. Dawson, Radiation background studies for the Phase-II Inner Tracker Upgrade, ATL-UPGRADE-PUB-2013-012 (2013).

[7] A. Holmes-Siedle and L. Adams, Handbook of radiation effects, $2^{\text {nd }}$ edition, Oxford University Press (2002).

[8] M. Citterio, S. Rescia and V. Radeka, A study of low noise JFETs exposed to large doses of gamma rays and neutrons, IEEE NSS/MIC 2 (1992) 794.

[9] M. Citterio, S. Rescia and V. Radeka, Radiation effects at cryogenic temperatures in Si-JFET, GaAs MESFET, and MOSFET devices, IEEE Trans. Nucl. Sci. 42 (1995) 2266.

[10] J. Grant et al., Wide bandgap semiconductors for harsh radiation environments, Nucl. Instrum. Meth. A 546 (2005) 213.

[11] http://www.semiconductor-today.com/news_items/2012/OCT/SEMISOUTH_241012.html. 